\title{
Determination of the Scenarios for the Future of Materials Technology
}

\author{
Astelio Silvera Sarmiento ${ }^{1 *}$, Luis Fernando Garcés Giraldo ${ }^{2}$ and Jovany Sepúlveda-Aguirre ${ }^{3}$ \\ ${ }^{1}$ National Research Vice President, Corporación Universitaria Americana, Barranquilla - Colombia \\ ${ }^{2}$ Researcher, Corporación Universitaria Americana, Medellín - Colombia \\ ${ }^{3}$ Vice President for Research, Corporación Universitaria Americana, Medellín - Colombia \\ ${ }^{*}$ Corresponding author
}

\begin{abstract}
Introduction. In the world the prospective studies have been taking great acceptance, especially in developing countries, as they allow the prioritization and targeting of efforts in research on a specific topic. Objective. Contrast the positions of the key members of 2 Colombian universities about the issues prioritized by a group of experts in relation to the materials technology. Materials and methods. The study of future scenarios was carried out through the delivery of the results of a prospective study, which conducted a survey of three groups belonging to 2 universities, Group 1. Leaders of the research groups with projects and lines of research in materials, Group 2. Teachers researchers that perform actions in these groups. Group 3. University managers. Results. Set the perceptions of respondents compared to the vision of the future for the technologies related to materials. Conclusions. It is important this exercise due to their results that can make possible the construction of a research agenda, in addition to other activities to address the challenges that researchers will face in materials technology.
\end{abstract}

Keywords-prospective; materials technology; perspectives; research programs

\section{INTRODUCTION}

To analyze the different areas where the global research systems are, it is important to consider what has happened in the countries that are shown as powers or developed, in addition to reviewing what happen with universities, centers and institutes that have carried high-impact research which enabled them to have a reputation in the international arena, it was found that the construction of the so-called knowledge societies has demanded of various instruments that come together in different ways such as: mental and conceptual maps, which set the installed capacity in research of each agency, in addition, its thematic areas of interest, improving the monitoring of the new trends and developments, which as a whole, allows you to observe, and redirect the strategic axes, and policies in research.

On the other hand, Colombia and the whole of their academic and scientific institutions, have limited information systems that allow to establish strategies in research in the institutional and geographical areas, which will serve as a tool to define policies and guidelines for the long-term research, in addition to the decision-making based on objective knowledge, as a space for ongoing management of knowledge that will help to establish projections for the short, medium and long term and clearly aligned to those of the country. The above does not attempt to put aside some exercises that have been made, particularly in the last three decades by some State entities and institutions in particular, however, the results have not been the expected. For instance, the actions carried out by the National System of Science, Technology and Innovation of Colombia, led by Colciencias, which has led to some conditions, many times naively, for the framing and progress of the research groups in programs that leads and that were defined by this entity [1].

As another edge, and with the participation of public and private entities, some of the activities of prospective analysis using different methodologies, have been made to learn about the evolution and future of a specific subject [2], among these exercises, it has been able to demonstrate the efforts made by different universities to determine areas or key thematic and international interest and agendas or research programs, embodied in strategic plans and actions logically supported in Strategic Programs. Also, through the strategic information analysis of future scenarios of research, academic networks of support has been set with the intention of establishing joint agendas [3]. However, these actions, although they were established as strategies to the institutions, many times they didn't beyond the realm of the administrative, without having a concrete support of indicators that give account of their impact.

To clarify this issue of the lack of impact compared to what has been done, it is enough to review the instruments and the results of the prospective exercises, which leads to think in the restructuring of the strategies of vision for the future of the institutions in Colombia, being even more necessary to reflect on the dynamics of innovation research, the latter, the most important for the survival of any company or institution in the current changing, globalised world environment [4]. Therefore, it should be enhanced the capabilities that are mature and strengthen those that have not made progress in order to make long-term projections that let the country to plan for the future in the medium and long term in the field of science, technology and innovation, with particular emphasis placed on issues on which there are certain strengths as a country.

In response to the above, this article is intended to establish a contrast between the positions of key members of 2 Colombian Universities in relation to the issues prioritized 
by a group of experts in materials technology and the challenges in this topic that are displayed for the future.

\section{MATERIALS AND METHODS}

\section{A. Prospective Analysis Methodology}

Stage 1. Choice of Expert Authors and Trends

It was made a search in data bases of the authors with wide level of citation and recognition in materials technology, in the data obtained was made a filter of the authors with institutional affiliation of the institutions best scored in the Academic Ranking of World Universities - ARWU. After having the data of the authors, in total there were 11, it was proceeded to make a formal invitation to participate in a panel of experts, to carry out an exercise-oriented under the Delphi methodology, invitation was responded for 6 of the international authors.

Stage 2. Application of the Delphi Technique [5].

10 questions were formulated directly related to the following theme (an abbreviation was assigned to each one for later use):

1. Nanotechnology for the production of materials and manufacturing - NT-

2. Production materials technologies - TPM-

3. Properties of new nanostructured materials - PN-

4. Computational and engineering developments for the materials production - DCISM-

5. Manufacturing Processes (simulation and modeling) -

6. Technologies to reduce energy consumption in production - cer-

7. Technologies for the combination of materials in manufacturing - CMM-

8. Technologies for biodegradable and recyclable materials - MBC-

9. Technology for polymeric materials -MP-

10. Technology for the energy industry materials - MIE-

After defining the survey with 10 semi-structured questions, three rounds of questions were made among the 6 experts, performing filter to the most recurrent responses in order to define trends. The implementation of the survey had an approximate duration of 6 months.

Stage 3. Comparison survey of results with groups in universities

A survey was conducted in three groups belonging to 2 universities, one located in the Caribbean Region and another one located in the Andean region of Colombia:

Group 1. Leaders of the research groups with projects and lines of research in materials.
Group 2. Teachers researchers that perform actions in these groups.

\section{Group 3. University managers}

In the survey were compared the results obtained from the rounds of questions (thematic, trend and foci), to establish later and under the guidance of the leaders of research a research agenda. The exercise carried out at this stage was a road mappin in different work sessions.

\section{RESULTS}

To initially establish the perceptions evidenced by the group of experts consulted, in table $\mathbf{1}$ was related the issues prioritized and guiding shafts to consider for future research.

TABLE I. PRIORITY THEMES AND GUIDING SHAFTS PUNCTUATED BY EXPERTS (ROUND 1)

\begin{tabular}{|c|c|c|c|c|c|c|c|c|c|c|}
\hline Expert & \multicolumn{7}{|c|}{ Thematic qualification for round 1 of questions* } \\
\hline & NT & TPM & PN & DCI & SM & RCE & CMM & MBC & MP & MIE \\
\hline 1 & 5 & 2 & 4 & 3 & 3 & 4 & 3 & 4 & 5 & 4 \\
\hline 2 & 5 & 3 & 3 & 3 & 3 & 5 & 3 & 4 & 5 & 5 \\
\hline 3 & 5 & 2 & 4 & 2 & 2 & 3 & 3 & 5 & 3 & 5 \\
\hline 4 & 5 & 1 & 4 & 2 & 1 & 4 & 4 & 4 & 4 & 5 \\
\hline 5 & 5 & 2 & 4 & 3 & 2 & 4 & 5 & 2 & 4 & 5 \\
\hline 6 & 5 & 3 & 3 & 1 & 2 & 5 & 2 & 4 & 3 & 5 \\
\hline Total & $\mathbf{3 0}$ & $\mathbf{1 3}$ & $\mathbf{2 2}$ & $\mathbf{1 4}$ & $\mathbf{1 3}$ & $\mathbf{2 5}$ & $\mathbf{2 0}$ & $\mathbf{2 3}$ & $\mathbf{2 4}$ & $\mathbf{2 9}$ \\
\hline
\end{tabular}

*It was requested in order to qualify for 1 to 5 in accordance with the perception about the vision of the future of the sub-item, being 5 the highest score and 1 the lowest.

Source: Prepared by the authors.

In Table $\mathbf{1}$ are displayed trends related to the use of nanotechnology (NT - 30 and 23), PN - technologies for reduction of energy consumption (RCE - 25; MIE - 29) and use of biodegradable materials (MBC - 23), finding a smaller proportion of the issues related to computational developments (DCI - 14); modeling and simulation (SM 13).

This is how the issues that obtained a higher score to 20 were prioritized, in order to realize the second round of experts, this time sending in the survey items as shown in Table 2 , with an indication of establish a trend in front of 6 themes with a higher score than indicated.

The second round of questions denotes a wide trend toward 2 themes that are being addressed by a number of research and are related to nanotechnology (NT - 28) technologies for reduction of energy consumption (RCE - 27; MIE - 27) and use of biodegradable materials (MBC - 27). With these answers the 4 highest scores are taken to define the perspective of the topic, being necessary in the Round 3 (Table 3) that the experts can define some key sub-themes to carry out research projects to respond to these trends. 
TABLE II. PRIORITY THEMES AND GUIDING SHAFTS PUNCTUATED BY EXPERTS (ROUND 2)

\begin{tabular}{|c|c|c|c|c|c|c|c|}
\hline Expert & \multicolumn{6}{|c|}{ Thematic qualification for round 2 of questions* } \\
\hline & NT & PN & RCE & CMM & MBC & MP & MIE \\
\hline 1 & 5 & 4 & 5 & 3 & 5 & 4 & 5 \\
\hline 2 & 4 & 4 & 5 & 2 & 3 & 4 & 5 \\
\hline 3 & 5 & 4 & 4 & 4 & 5 & 3 & 3 \\
\hline 4 & 4 & 3 & 5 & 4 & 5 & 3 & 5 \\
\hline 5 & 5 & 4 & 4 & 3 & 4 & 3 & 4 \\
\hline 6 & 5 & 5 & 4 & 4 & 5 & 3 & 5 \\
\hline Total & $\mathbf{2 8}$ & $\mathbf{2 4}$ & $\mathbf{2 7}$ & $\mathbf{2 0}$ & $\mathbf{2 7}$ & $\mathbf{2 0}$ & $\mathbf{2 7}$ \\
\hline
\end{tabular}

* It was requested in order to qualify for 1 to 5 in accordance with the perception about the vision of the future of the sub-item, being 5 the highest score and 1 the lowest.

Source: Prepared by the authors.

TABLE III. PRIORITY THEMES AND GUIDING SHAFTS PUNCTUATED BY EXPERTS (ROUND 3)

\begin{tabular}{|c|c|c|c|c|}
\hline Expert & \multicolumn{4}{|c|}{ Thematic qualification for round 3 of questions* } \\
\hline & NT & RCE & MBC & MIE \\
\hline 1 & 5 & 5 & 5 & 5 \\
\hline 3 & 5 & 5 & 3 & 5 \\
\hline 4 & 5 & 4 & 3 & 5 \\
\hline 5 & 5 & 5 & 3 & 5 \\
\hline 6 & 5 & 5 & 4 & 4 \\
\hline Total & $\mathbf{3 0}$ & $\mathbf{2 8}$ & $\mathbf{2 3}$ & $\mathbf{2 9}$ \\
\hline
\end{tabular}

*It was requested in order to qualify for 1 to 5 in accordance with the perception about the vision of the future of the sub-item, being 5 the highest score and 1 the lowest.

Source: Prepared by the authors.

According to the results of the rounds 1,2 and 3 of questions, a report of trends and prospects for the topic of materials technology was made, report that was distributed among 3 groups of 2 universities, distributed in the following manner: Group 1. Leaders of the research groups with projects and lines of research in materials, Group 2. Teacher researchers that perform actions in these groups. Group 3. University managers.

After making the socialization of the report, it proceeded to contrast and do the validation of the results with the selected groups, for this purpose, it was conducted an exercise of road mapping [6] for the technology of materials, each session with a duration of approximately 4 hours. In the first session were contrasted the market components, products and technology for the 10 subjects evaluated by experts (Table 4).

In the exercise of the Table 4, it is noticeable the importance that is given to the technological component (39) in the future of the materials technology, followed by the products item (37) and in the last place of the scale the market (34).
TABLE IV. EXERCISE ROAD MAP PRIORITY TOPICS

\begin{tabular}{|c|c|c|c|}
\hline Topic & \multicolumn{3}{|c|}{ EVALUATED COMPONENT ROAD MAP } \\
\hline & Market & Products & Technology \\
\hline NT & 4 & 5 & 5 \\
\hline TPM & 3 & 3 & 2 \\
\hline PN & 4 & 4 & 5 \\
\hline DCI & 3 & 3 & 5 \\
\hline SM & 2 & 2 & 2 \\
\hline RCE & 4 & 4 & 5 \\
\hline CMM & 2 & 3 & 2 \\
\hline MBC & 4 & 4 & 4 \\
\hline MP & 3 & 5 & 4 \\
\hline MIE & 5 & 4 & 39 \\
\hline Total & $\mathbf{3 4}$ & $\mathbf{3 7}$ & 5 \\
\hline
\end{tabular}

* It was requested in order to qualify for 1 to 5 in accordance with the perception about the vision of the future of the sub-item, being 5 the highest score and 1 the lowest.

TABLE V. EXERCISE ROAD MAP PRIORITY TOPICS IN THE SECOND ROUND OF EXPERTS

\begin{tabular}{|c|c|c|c|}
\hline \multirow{2}{*}{ Topic } & \multicolumn{4}{|c|}{$\begin{array}{c}\text { EVALUATED COMPONENT ROAD MAP (2nd } \\
\text { round) }\end{array}$} \\
\hline & Market & Products & Technology \\
\hline NT & 2 & 4 & 5 \\
\hline PN & 3 & 5 & 5 \\
\hline RCE & 2 & 5 & 5 \\
\hline CMM & 3 & 3 & 2 \\
\hline MBC & 3 & 5 & 5 \\
\hline MP & 3 & 3 & 5 \\
\hline MIE & 2 & 5 & 5 \\
\hline Total & $\mathbf{1 8}$ & $\mathbf{3 0}$ & $\mathbf{3 2}$ \\
\hline
\end{tabular}

* It was requested in order to qualify for 1 to 5 in accordance with the perception about the vision of the future of the sub-item, being 5 the highest score and 1 the lowest.

In a new session of road map was applied the same logic to the results obtained in the second round of questions to the experts. The results are presented in table 5.

In this new exercise is evident again the preponderance that has the technology, without forgetting that the final products (quality, materials, alloys and combinations) was an important finding for the definition of a research program that respond to the findings of the set of exercises here exposed.

\section{DISCUSSION}

The results obtained in the issues prioritized by the experts in rounds 1, 2 and 3 correspond to the exposed by [7], which demonstrate the importance of issues such as simulation and modeling in nanomaterials, nanotechnologies for processing and manufacturing, technologies for measurement and nano-analysis, technology NEMS, synthesis and processing technologies in materials, nanodevices and sensors, new materials of control structure in nanonivel, materials in the environment and energy, Nanobiology, Nanoscience for a safe society, among other 
findings. Also, the results of the exercises are compatible with the established by the Superior Council of Scientific Research (Madrid) [8], which makes mention of some of the materials technologies for the future, these are: advanced ceramic materials, new advanced metallic, polymer materials, magnetic materials, nanomaterials, biomaterials, materials for laser, energy and the environment.

\section{CONCLUSIONS}

The foresight exercises are important due to the view of the changes and trends that have a specific topic that could positively or negatively affect the development of a technology. In this way, and with the combination of different tools the institutional capacities are being strengthened to anticipate the future.

Nanotechnology is a fundamental axis that moves the industry and materials technology; in addition, it must be the subject of discussion of the research groups of participating groups in this study, in order to establish a concrete agenda for research that respond to the challenges of the industry, in this topic, that each day are increasing.

The energy industry is increasingly demanding different technologies, materials and supplies, and research groups are called to anticipate trends, with plans, programs and projects in a clear and precise agenda of support in research.

\section{ACKNOWLEDGMENTS}

The authors would like to thank Corporación Universitaria Americana and to the Fondo para el Desarrollo de la Investigación of the Corporación Universitaria Lasallista for providing the resources for the realization of this research.

\section{REFERENCES}

[1] J. Rodríguez. "Informe PUI de Energía. Lugar de los PUI dentro del contexto actual de la investigación.”. Bogotá: PIU - Seminario Investigaciones en Red. 1999.

[2] C. Cagnin; A. Hayas \& O. Saritas. "Future-oriented technology analysis: Its potential to address disruptive transformations". Technological Forecasting and Social Change, Vol. 80, Num 3, 2013. 379-385. doi.org/10.1016/j.techfore.2012.10.001

[3] C. Duque; M. Brijaldo. R. Molina. "Programas estratégicos: un reto institucional para la Universidad en el siglo XXI”. Bogotá: TM editores e impresores. 2001.

[4] J. Sepúlveda; L. Garcés; J. Serrano y H. Gómez An approach between tools for the analysis of tendencies and business innovation management. Revista Lasallista de Investigación, Vol. 13, num 2, 2106. 178- 187. DOI: $10.22507 /$ rli.v13n2a16

[5] E. Astigarraga. El método Delphi. Bilbao, España: Universidad de Deusto, Facultad de CC.EE. y Empresariales. 2009.

[6] R. Phaal; C. Farrukh; \& D. Probert. "Technology roadmapping - a planning framework for evolution and revolution”. Technological Forecasting and Social Change, Vol. 71, 2004 . pp. 5-26

[7] N. Su; \& P. Lee. "Future perspectives on nanotechnology/material development: Delphi studies and Sci-Tech policies in Japan, Mainland China and Taiwan”. 2008. (pp. 2266-2272). China: IEEE. doi:10.1109/PICMET.2008.4599849

[8] C. Mijangos. J. Moya. "Nuevos materiales en la sociedad del siglo XXI” España, Consejo Superior de investigaciones científicas 